February, 2002

\title{
The Wilson-Polchinski Renormalization Group Equation in the Planar Limit
}

\author{
C. Becchi 用, S. Giusto [ and C. Imbimbo f \\ Dipartimento di Fisica, Università di Genova \\ and \\ Istituto Nazionale di Fisica Nucleare, Sezione di Genova \\ via Dodecaneso 33, I-16146, Genoa, Italy
}

\begin{abstract}
We derive the Wilson-Polchinski RG equation in the planar limit. We explain that the equation necessarily involves also non-planar amplitudes with sphere topology, which represent multi-trace contributions to the effective action. The resulting RG equation turns out to be of the Hamilton-Jacobi type since loop effects manifest themselves through terms which are linear in first order derivatives of the effective action with respect to the sources. We briefly outline applications to renormalization of non-commutative field theories, matrix models with external sources and holography.
\end{abstract}

${ }^{*}$ E-Mail: becchi@ge.infn.it

${ }^{\dagger}$ E-Mail: giusto@ge.infn.it

${ }^{\ddagger}$ E-Mail: imbimbo@ge.infn.it 


\section{Introduction}

Planar field theories introduced years ago in the seminal work of 't Hooft [1] have recently enjoyed renewed interest in connection with holography [2], [3] and noncommutative field theory [4] (for a review and references see [5]).

The holographic conjecture relates $S U(N)$ (supersymmetric) gauge theory in $d$ dimensions to string theory in $(d+1)$-dimensional anti de Sitter space. Consider a $(d+1)$-dimensional ball $B_{d+1}$ in the interior of AdS space, whose boundary $S_{d}$ is a $d$-dimensional sphere homotopic to the AdS boundary. The action of AdS supergravity evaluated on the solution of the classical field equations with boundary values prescribed on $S_{d}$ and integrated on $B_{d+1}$ is a functional of the field boundary values: Maldacena's conjecture identifies this functional with the generating functional of correlators of gauge-invariant composite operators of the $S U(N)$ theory in the large $N$ limit and large 't Hooft gauge coupling, renormalized at a scale which is related to the radial distance of the sphere $S_{d}$ from the AdS boundary.

This intriguing correspondence between the AdS radial coordinate and the renormalization scale of the gauge theory has suggested to some authors [8] the possibility that the Wilson-Polchinski renormalization group equation of the $S U(N)$ quantum field theory in the large $N$ limit might correspond to the classical equations of motion of the supergravity theory. The RG Wilson-Polchinski equation [6], [7] is a differential equation for the effective action of first order in the renormalization scale: therefore, in the scenario proposed in [8], it is put in relation with the supergravity field equations written in the Hamilton-Jacobi form, with the AdS radial direction identified with the time coordinate. Since radial slices are not space-like surfaces in AdS, this identification appears problematic. At any rate, any attempt to make this proposal more concrete requires the knowledge of the large $N$ limit of the RG Wilson-Polchinski equation. This is what we do in this article.

The RG Wilson-Polchinski equation in the planar limit might also be of interest in the study of the renormalization properties of the non-commutative quantum field theories which emerge in a certain limit of open string theory. It has in fact been observed [4] that the perturbative expansion for these theories reduces, in the limit of large non-commutative parameter $|\Theta|$, to planar diagrams. It is therefore relevant to understand if a RG equation involving only planar diagrams can be written for such theories.

Let us comment on the meaning of planar diagrams in the context of field theories discussed in [4], whose non-commutative structure is described by the Moyal product. Feynman diagrams of non-commutative theories have two equivalent descriptions: in one description propagators are single lines and lines coming out of vertices are ordered. In the other description propagators are double lines carrying opposite orientations. In both pictures, a Feynman diagram uniquely determines a Riemann surface on which it can be drawn: this surface is oriented, due to the orientation of 


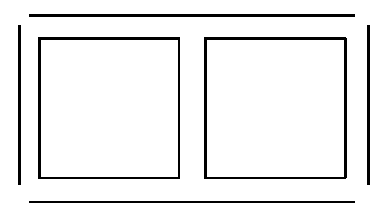

(a)

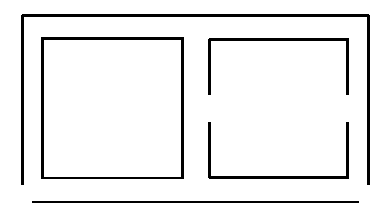

(b)

Figure 1: Planar (a) and non-planar (b) diagrams in non-commutative theory

propagator lines, and it is closed, i.e. without boundaries, as long as one restricts oneself to theories without single lines (quarks). We will always refer to the double line picture in the following. In this picture diagrams corresponding to amputated and connected Green functions are called planar if they can be drawn on the sphere and if all the external legs are attached to the same line. Figure 1 shows two examples of planar $(a)$ and non-planar (b) diagrams in non-commutative theory with cubic interaction, both of which can be drawn on a sphere: in this figure external legs are represented by breaks in the propagator lines. With this definition, non-planar diagrams like $(b)$ are exponentially suppressed in Moyal non-commutative theories in the limit of large non-commutativity (see Appendix A). The effective action appearing in the RG group equation is the generating functional of amputated connected Green functions. It is relatively easy to convince oneself and it will be explained in detail in Section 1 that one cannot write a RG group equation involving exclusively planar amplitudes: diagrams like (b) must be included in the "planar" Wilson-Polchinski equation.

In $S U(N)$ matrix theories sphere diagrams like $(a)$ correspond to terms in the Wilsonian effective action which are single traces of the matrix source while diagrams like $(b)$ are associated to multi-traces. The large $N$ limit for the Wilsonian action is to be taken by keeping fixed both the 't Hooft couplings and the normalized trace invariants of the external matrix source. In this limit sphere diagrams - both of (a) and (b) type - are of the same (leading) order in the $1 / N$ expansion. This makes clear why the planar limit of RG equation that we derive describes all sphere diagrams, irrespectively of where the external legs are attached.

This paper is organized as follows: in Section 2 a diagrammatic derivation of the planar RG equation (8) for 0-dimensional non-commutative theory is presented. It is interesting that this equation, unlike the usual RG Wilson-Polchinski equation, is of the Hamilton-Jacobi type. The first derivative with respect to the renormalization scale of the Wilsonian action equals a sum of two terms: the first is the classical one and is quadratic in the field derivatives of the effective action. The second term, which encodes the quantum corrections, is linear in field derivatives of the action: 
this is in contrast with the usual RG equation for which the quantum term involves second order field derivatives of the action. In Section 3 the same equation is derived by functional methods, by taking the large $N$ limit of the Wilson-Polchinski equation for scalar $N \times N$ matrix models. In Section 4 a formulation of the equation in terms of eigenvalues of the matrix source is also given: in these variables the planar $\mathrm{RG}$ equation is the Hamilton-Jacobi equation of a system of $N$ free particles in 1 dimension. This reformulation leads to a compact expression for the effective action in the large $N$ limit. In Section 5 we write down the planar RG equation for a scalar matrix theory in $d$ dimensions. Finally in Section 6 we state our conclusions.

In the work of Ref. [10] a large $N$ differential equation for the generating functional of the correlators of single-trace composite operators of a zero-dimensional matrix model is derived. This generating functional might be thought of as the zerodimensional analogue of the on-shell supergravity action which enters the holographic conjecture in the formulation of [3]. In Appendix B we rederive this equation in a language which is closer to the one of the present paper and at the same time we clear up a few imprecisions present in the equation of [10]. It is important however to emphasize that this equation is not an equation for the Wilson-Polchinski effective action and, if generalized to higher dimensions, it does not encode the renormalization properties of the corresponding quantum field theory.

\section{The "Planar" RG Equation from Feynman Dia- grams}

Let us consider a $d$-dimensional scalar field theory, whose field $\phi(x)$ takes values in some non-commutative space. We can think of this space either as the space of $N \times N$ hermitian matrices or as the non-commutative algebra associated with the Moyal product. Let $h_{t, t_{0}}^{\text {planar }}\left(p_{1}, \ldots, p_{n}\right)$ be the planar contribution to the $n$-point connected and amputated Green function computed with a regulated propagator $\Delta_{t, t_{0}}(x, y)$ which depends both on the ultra-violet scale $t_{0} \equiv \Lambda_{0}^{-2}$ and the running (infra-red) scale $t \equiv \Lambda^{-2}$. As we explained in the introduction $h_{t, t_{0}}^{\text {planar }}\left(p_{1}, \ldots, p_{n}\right)$ receives contributions from diagrams of type $(a)$ of Figure 1 .

The Wilson-Polchinski RG equation describes the dependence on the infra-red scale $t$ of the generating functional $H_{t}[\varphi]$ of (both planar and non-planar) regulated, amputated and connected Green functions. Since the dependence of the Green functions on the ultra-violet cut-off $t_{0}$ never enters the discussion, henceforth we will drop explicit reference to $t_{0}$. One might hope that a consistent truncation of the WilsonPolchinski equation to planar Green functions would lead to an evolution equation 
for the planar functional generator $H_{t}^{\text {planar }}[\varphi]$

$$
H_{t}^{\text {planar }}[\phi] \equiv \sum_{n=1}^{\infty} \frac{1}{n} \int \prod_{i=1}^{n} d^{d} p_{i} \delta\left(\sum_{j=1}^{n} p_{i}\right) h_{t}^{\text {planar }}\left(p_{1}, \ldots, p_{n}\right)\left\langle\varphi\left(p_{1}\right) \ldots \varphi\left(p_{n}\right)\right\rangle
$$

where $\langle\cdot\rangle$ is the trace on the non-commutative algebra. We will now explain why such a truncation does not exist: in order to write down a "planar" RG equation one must extend the functional in Eq. (1) to include multi-trace terms.

Let us recall the diagrammatic interpretation of the ordinary (commutative) RG equationf. The $t$-derivative of $H_{t}$ is the formal sum of all the diagrams obtained from those contributing to $H_{t}$ by replacing one of the internal propagators $\Delta_{t}$ with its derivative $\dot{\Delta}_{t}$, i.e. by inserting $\dot{\Delta}_{t}$ between the new external legs created after cutting the propagator line. This leads to the diagrammatic equation displayed in Figure 2 . In this picture a box with a label $\alpha$ on the lower right-hand side corner represents an amputated connected Green function with $\alpha$ external legs. A crossed line denotes the insertion of the $\dot{\Delta}_{t}$ factor. The two diagrams on the r.h.s. of the equation correspond to the two possible cases: in the first case the removed propagator line lies on a tree line of the skeleton graph while in the second case the propagator belongs to a loop.

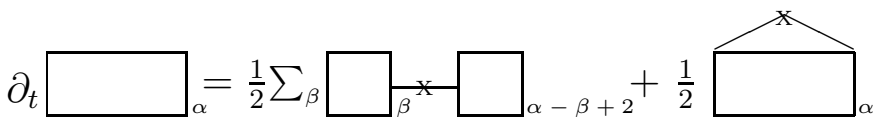

Figure 2: The ordinary RG evolution equation

Considering now the case of non-commutative or matrix theories, planar diagrams in the double-line picture have a preferred line, that will be called peripheral. This is the line to which all the external legs are attached: external legs of planar diagrams have therefore a natural cyclic order. Consider now the second term on the r.h.s. of the diagrammatic equation in Figure 2: when the external legs of a planar amplitude which are joined by the $\dot{\Delta}_{t}$ are not contiguous, this term induces a non-vanishing contribution to the $t$-derivative of non-planar amplitudes. These are special nonplanar amplitudes, like (b) of Figure 1: they have sphere topology but have external legs attached to more than one single line. Let us name holes the lines, other than the peripheral line, to which external legs are attached. It is clear now that if we want to write a RG equation for the planar graphs we must, for consistency, include also all the sphere graphs with any number of holes to which an arbitrary number of external lines are attached. We might call these graphs swiss-cheese (SC) for their resemblance to swiss-cheese slices when drawn on a plane.

\footnotetext{
${ }^{1}$ Notice that, in order to avoid alternating signs, we have adopted a sign convention which is opposite to the standard one.
} 
Let us picture an $\alpha$-point planar, connected and amputated amplitude with a box, whose perimeter represents the peripheral line labelled by an index $\alpha$ on the right-hand side lower corner. SC amplitudes with $n$ holes, $\alpha_{0}$ external legs attached to the peripheral line and $\alpha_{i}$ external legs attached to the $i$-th hole (with $i=1, \ldots, n$ ) are represented by a box with $n$ internal circular holes: the index $\alpha_{0}$ is associated with the perimeter of the box while $\alpha_{i}$ 's label the internal circles. Two examples are depicted in Figure 3. Note that we call amplitude the sum of all diagrams with the same structure of external legs. Therefore the interior of boxes in Figure 3 should be thought as filled with all possible nets of propagators. Propagators will be now double lines and insertions of $\dot{\Delta}_{t}$ will be indicated by crosses: $\approx$. Let us also distinguish two kinds of propagators - external and internal. External propagators have one line belonging to the peripheral line, internal propagators are all the others.

Given these graphical rules, the diagrammatic RG equation for SC amplitudes which replaces Figure 2 is displayed in Figure 4 . The four terms on the r.h.s. of this equation correspond to the four possible positions of the removed propagator inside the graph. In the first and the second term the cut propagator connects two otherwise disconnected graphs; in the third and fourth term removing the propagator leaves the diagram connected. The removed propagator of terms one and three is external, that of terms two and four is internal.

Terms one and two on the r.h.s. of the equation in Figure 1 replace the first (classical) term on the r.h.s. of the ordinary RG equation in Figure 2. Terms three and four correspond to the loop term of ordinary RG flow (the last term of Figure 2). It is the third term on the r.h.s. of the RG equation in Figure that generates non-planar diagrams starting from planar ones.

We now want to translate the graphical "planar" RG equation in Figure 1 into analytic form. This requires the introduction of a functional generator for SC amplitudes generalizing the one for planar amplitudes given in Eq. (1). As Eq. (11) shows, planar amplitudes are represented in the effective action by a single trace of the product of the sources. Likewise, each hole of a SC amplitude is associated with the trace of the product of as many sources as legs attached to the hole. Hence, SC non-planar amplitudes are associated with multi-traces, one trace for each hole and one trace for the peripheral line. It is then clear that, when writing down the effective action, there is no distinction between holes and peripheral line: we can

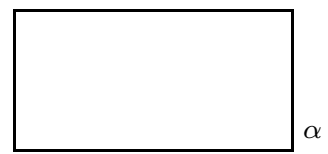

(a)

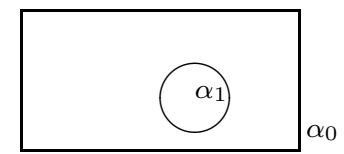

(b)

Figure 3: SC diagrams with 0 (a) and 1 (b) holes 

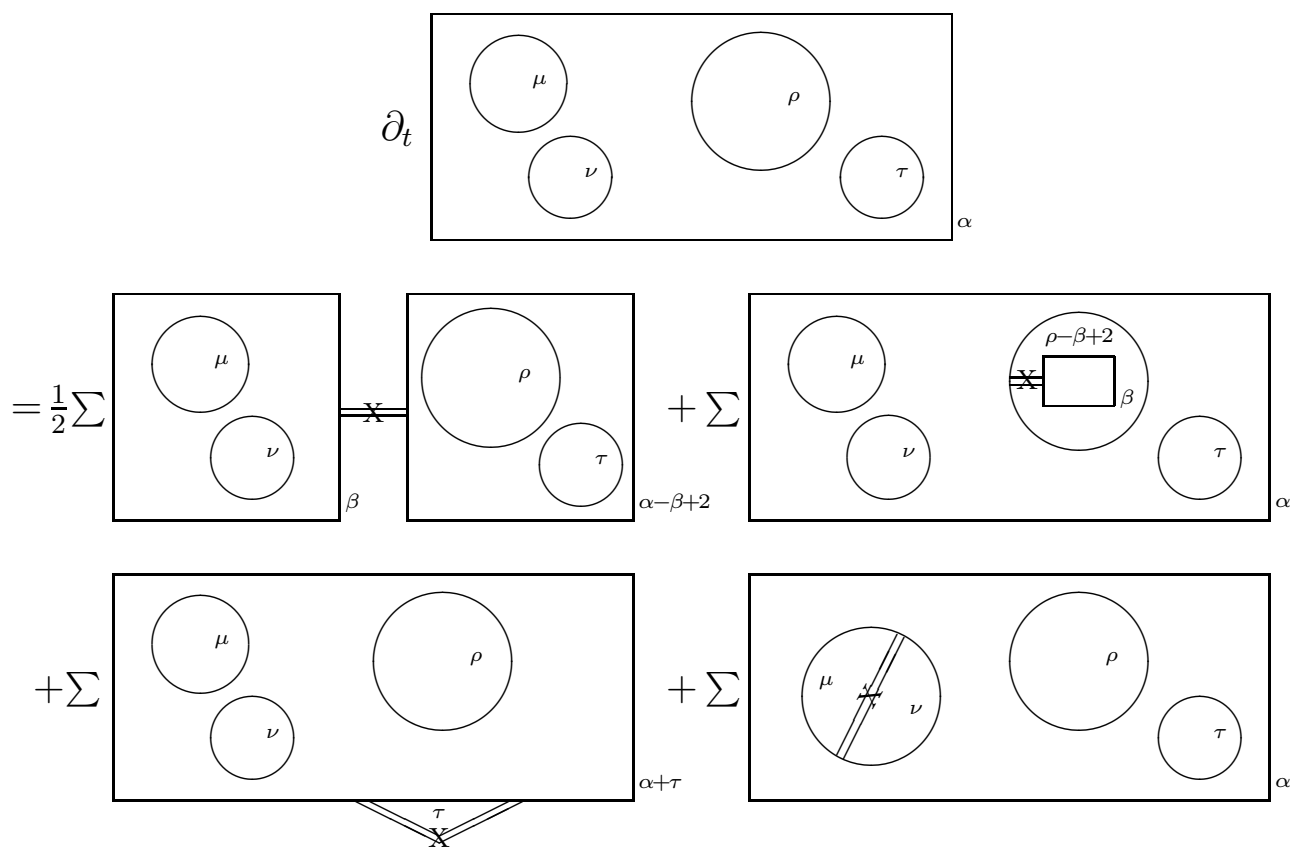

Figure 4: The "planar" RG equation

characterize the SC amplitude by the number $m_{k}$ of traces of products of $k$ sources without the need to distinguish the trace associated with the peripheral line from the traces associated with the holes. Of course diagrammatically this corresponds to the fact that SC graphs can be drawn on a sphere where the peripheral line is just another hole. Henceforth we will refer to SC amplitudes when drawn on a sphere as multi-trace amplitudes. More explicitely, if we call $f_{t}\left(n,\left\{m_{k}\right\}\right)$ the SC amplitude with $n$ peripheral legs and $m_{k} k$-leg holes and $h_{t}\left(\left\{m_{k}\right\}\right)$ the multi-trace amplitude with $m_{k} k$-leg holes — we are suppressing explicit reference to external momenta for notational simplicity — then one has

$$
\frac{1}{n} f_{t}\left(n,\left\{m_{k}\right\}\right)=\left(m_{n}+1\right) h_{t}\left(\left\{m_{k}+\delta_{k, n}\right\}\right)
$$

If we represent multi-trace amplitudes with spheres with holes the RG equation in Figure 1 becomes the graphical equation of Figure 5. Since the distinction between internal and external propagators is immaterial when we draw the diagram on the sphere, the first and the last pair of terms on the r.h.s. of the equation in Figure 4 become respectively the first and the second term in the equation of Figure 5. When the theory is a $N \times N$ matrix theory, the sphere representation of the RG equation in Figure 5 is perhaps more natural, since in the $1 / N$ expansion sphere diagrams with holes are precisely the leading $\left(O\left(N^{2}\right)\right)$ contribution to the effective Wilsonian action. 


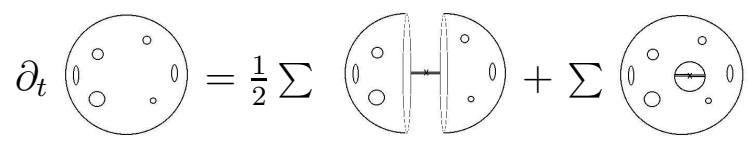

Figure 5: The "planar" RG equation on the sphere

In the next section where we will derive the "planar" RG equation by functional methods we will adopt the matrix point of view.

Let $p_{k, j_{k}}^{\left(i_{k}\right)}$ be the $j_{k}$-th momentum (with $j_{k}=1, \ldots, k$ ) which flows into the $i_{k}$ hole (with $i_{k}=1, \ldots, m_{k}$ ) of a multi-trace graph corresponding to the connected amputed amplitude $h_{t}^{\left\{m_{k}\right\}}\left(p_{k, j_{k}}^{\left(i_{k}\right)}\right)$. The Wilsonian effective action writes

$$
H_{t}[\varphi] \equiv \sum_{\left\{m_{k}\right\}} \int_{k, i_{k}, j_{k}} d p_{k, j_{k}}^{\left(i_{k}\right)} \delta\left(\sum_{k, i_{k}, j_{k}} p_{k, j_{k}}^{\left(i_{k}\right)}\right) h_{t}^{\left\{m_{k}\right\}}\left(p_{k, j_{k}}^{\left(i_{k}\right)}\right) \prod_{k} \prod_{i_{k}=1}^{m_{k}}\left\langle\varphi\left(p_{k, 1}^{\left(i_{k}\right)}\right) \cdots \varphi\left(p_{k, k}^{\left(i_{k}\right)}\right)\right\rangle
$$

To simplify our task and notation we shall disregard momentarily the momenta, therefore limiting our analysis to zero-dimensional non-commutative field theory. Obviously in this case the propagator is simply a constant which we will identify with the RG scale $t$ and $\dot{\Delta}_{t}$ reduces to 1 . The contribution of a given diagram to the corresponding amplitude is just the product of the combinatorial factor of the diagram with the required powers of the couplings and of the propagator. It should be clear however that the structure of the RG equation - which is captured by the graphical Equations Figures 4 , 5 - is independent of the dimension of space-time. In Section 5 we will explicitly write down the $d$-dimensional version of the RG equation.

In 0 dimensions the functional generator in Eq. 3 becomes

$$
H_{t}\left(\left\{y_{k}\right\}\right) \equiv \sum_{\left\{m_{k}\right\}} h_{t}\left(\left\{m_{k}\right\}\right) \prod_{k=1}^{\infty} y_{k}^{m_{k}}
$$

where

$$
y_{k} \equiv\left\langle\varphi^{k}\right\rangle
$$

The first term on the r.h.s of the diagrammatic equation in Figure 5 corresponds to the following contribution to $\partial_{t} H_{t}\left(\left\{y_{k}\right\}\right)$

$$
\begin{aligned}
& \frac{1}{2} \sum_{k, k^{\prime}=1}^{\infty} \sum_{\left\{m_{p}\right\}} \sum_{\left\{m_{p}^{\prime}\right\}} k m_{k} k^{\prime} m_{k^{\prime}}^{\prime} h_{t}\left(\left\{m_{p}\right\}\right) h_{t}\left(\left\{m_{p}^{\prime}\right\}\right) \frac{y_{k+k^{\prime}-2}}{y_{k} y_{k^{\prime}}} \prod_{p=1}^{\infty} y_{p}^{m_{p}+m_{p}^{\prime}} \\
& =\frac{1}{2} \sum_{k, k^{\prime}=1}^{\infty} k k^{\prime} y_{k+k^{\prime}-2} \partial_{y_{k}} H_{t} \partial_{y_{k^{\prime}}} H_{t}
\end{aligned}
$$

The summand on the l.h.s. of the equation above corresponds to the joining of two multi-trace diagrams labelled respectively by the sequences $\left\{m_{p}\right\}$ and $\left\{m_{p}^{\prime}\right\}$; 
the crossed propagator connecting the two diagrams goes from a hole of the first diagram with $k$ external legs to a hole of the second diagram with $k^{\prime}$ legs. The factor $k m_{k} k^{\prime} m_{k^{\prime}}^{\prime}$ counts the different ways of joining two such diagrams. The resulting connected multi-trace diagram has a hole with $k+k^{\prime}-2$ legs replacing the two holes with $k$ and $k^{\prime}$ legs: this is accounted for by the $\frac{y_{k+k^{\prime}-2}}{y_{k} y_{k^{\prime}}}$ factor. The overall $\frac{1}{2}$ factor is due to the fact that exchanging the two multi-trace diagrams produces the same connected amplitude.

The second term on the r.h.s of the diagrammatic equation in Figure 5 yields:

$$
\begin{aligned}
& \frac{1}{2} \sum_{k=1}^{\infty} \sum_{i=0}^{k-2} \sum_{\left\{m_{p}\right\}} k m_{k} h_{t}\left(\left\{m_{p}\right\}\right) \frac{y_{i} y_{k-2-i}}{y_{k}} \prod_{p=1}^{\infty} y_{p}^{m_{p}} \\
& =\frac{1}{2} \sum_{k=1}^{\infty} \sum_{i=0}^{k-2} k y_{i} y_{k-2-i} \partial_{y_{k}} H_{t}
\end{aligned}
$$

The summand in the l.h.s. of Eq. (77) describes the process of joining two legs of the same hole of a multi-trace diagram labelled by the sequence $\left\{m_{p}\right\}$. Let $k$ be the number of external legs of this hole. The possible resulting multi-trace diagrams have one less hole with $k$ legs and two more holes with $i$ and $k-2-i$ legs respectively, where $i=0, \ldots, k-2$. This explains the $\frac{y_{i} y_{k-2-i}}{y_{k}}$ factor. Note that when $i=0$ and $i=k-2$ one of the two holes has no external legs attached to it: if we define $y_{0}=1$, the factor $\frac{y_{i} y_{k-2-i}}{y_{k}}$ is the correct one for these cases too. Given $k$, there are $k m_{k}$ ways to choose one of the two legs to be joined; the inequivalent choices for the second leg are labelled by the integer $i$ with $i=0, \ldots,\left[\frac{k-2}{2}\right]$. Since the factor $\frac{y_{i} y_{k-2-i}}{y_{k}}$ is invariant under the substitution $i \rightarrow k-2-i$, we can equivalently sum over $i$ in the range $[0, k-2]$ and divide by $\frac{1}{2}$.

In conclusion the $\mathrm{RG}$ equation for the functional generator of multi-trace amplitudes in 0 dimensions reads:

$$
\partial_{t} H_{t}=\frac{1}{2} \sum_{k, k^{\prime}=1}^{\infty} k k^{\prime} y_{k+k^{\prime}-2} \partial_{y_{k}} H_{t} \partial_{y_{k^{\prime}}} H_{t}+\frac{1}{2} \sum_{k=1}^{\infty} \sum_{i=0}^{k-2} k y_{i} y_{k-2-i} \partial_{y_{k}} H_{t}
$$

The striking feature of this RG equation is that only first derivatives with respect to the sources $y_{k}$ appear: thus the equation is of the Hamilton-Jacobi type. This is to be contrasted with the Wilson-Polchinski equation for ordinary (commutative) field theory which contains second order derivatives with respect to the sources. For example, the ordinary RG equation of Figure 2 for a scalar field in 0 dimensions reads

$$
\partial_{t} H_{t}=\frac{1}{2}\left(\partial_{\varphi} H_{t}\right)^{2}+\frac{1}{2} \partial_{\varphi}^{2} H_{t}
$$

where $H_{t}(\varphi)=\sum_{n=0}^{\infty} \frac{1}{n !} h_{t}(n) \varphi^{n}$ is the generating functional of connected amputated amplitudes $h_{t}(n)$ for the scalar field whose source is $\varphi$. The first "classical" term in 
the r.h.s. of the ordinary RG equation is of the same form of its analog in the noncommutative RG: the difference between the two equations lies in the "loop" term, which becomes first order in source derivatives when going to the non-commutative context.

A solution of the $\mathrm{RG}$ equation (8) is specified by giving the initial condition $\left.H_{t}\left(\left\{y_{k}\right\}\right)\right|_{t=0}$. This should be interpreted as the "bare" or classical interaction Lagrangian?, which is usually specified as a linear function of the $y_{k}$ 's. Note however that even if the initial condition is linear in the $y_{k}, H_{t}$ for $t \neq 0$ will necessary be non-linear: this reflects the impossibility of writing down the RG equation for the planar functional generator (11) that we have emphasized at the beginning at this section.

To consider a concrete example, let us choose the initial condition corresponding to a $\lambda\left\langle\phi^{4}\right\rangle$ theory:

$$
\left.H_{t}\left(\left\{y_{k}\right\}\right)\right|_{t=0}=\frac{1}{4} \lambda y_{4}
$$

Let us remark that when the intial condition is even under "parity" transformation $y_{k} \rightarrow(-1)^{k} y_{k}$ we can consistently truncate our equation to an effective action $H_{t}^{(+)} \equiv$ $\left.H_{t}\right|_{y_{2 k+1}=0}$ depending only on the even $y_{2 k}$ variables. This is due to the circumstance that the RG equation both conserves parity and is first order in $y_{k}$-derivatives. The truncated $\mathrm{RG}$ equation is

$$
\partial_{t} H_{t}^{(+)}=2 \sum_{k, k^{\prime}=1}^{\infty} k k^{\prime} y_{2 k+2 k^{\prime}-2} \partial_{y_{2 k}} H_{t}^{(+)} \partial_{y_{2 k^{\prime}}} H_{t}^{(+)}+\sum_{k=1}^{\infty} \sum_{i=0}^{2 k-2} k y_{2 i} y_{2 k-2-2 i} \partial_{y_{2 k}} H_{t}^{(+)}
$$

It is straightforward to compute the solution of Eq. (11) with initial condition (10) as perturbative expansion in $t$ :

$$
\begin{aligned}
& H_{t}^{(+)}\left(\lambda ;\left\{y_{k}\right\}\right)=\frac{1}{4} \lambda y_{4}+\left(\lambda y_{2}+\frac{1}{2} \lambda^{2} y_{6}\right) t+\left(\frac{3}{2} \lambda^{3} y_{8}+\frac{5}{2} \lambda^{2} y_{4}\right. \\
& \left.+\frac{3}{4} \lambda^{2} y_{2}^{2}+\frac{1}{2} \lambda\right) t^{2}+\left(\frac{11}{2} \lambda^{4} y_{10}+\frac{28}{3} \lambda^{3} y_{6}+\frac{9}{2} \lambda^{2} y_{2}+5 \lambda^{3} y_{2} y_{4}\right) t^{3} \\
& +\left(\frac{91}{4} \lambda^{5} y_{12}+\frac{165}{4} \lambda^{4} y_{8}+\frac{45}{2} \lambda^{3} y_{4}+21 \lambda^{4} y_{2} y_{6}+\frac{27}{2} \lambda^{3} y_{2}^{2}\right. \\
& \left.+\frac{75}{8} \lambda^{4} y_{4}^{2}+\frac{9}{8} \lambda^{2}\right) t^{4}+\left(27 \lambda^{3} y_{2}+\frac{27}{2} \lambda^{4} y_{2}^{3}+135 \lambda^{4} y_{2} y_{4}+126 \lambda^{4} y_{6}\right. \\
& \left.+84 \lambda^{5} y_{4} y_{6}+99 \lambda^{5} y_{2} y_{8}+\frac{1001}{5} \lambda^{5} y_{10}+102 \lambda^{6} y_{14}\right) t^{5}+O\left(t^{6}\right)
\end{aligned}
$$

\footnotetext{
${ }^{2}$ Due to our sign convention, the initial condition on $H_{t}$ is actually minus the interaction Lagrangian.
} 
The interested reader can verify that the coefficients of the single terms in this sum count the number of the corresponding Feynman diagrams multiplied by their combinatorial factor. Owing to the choice of the factor $\frac{1}{4}$ in the interaction Eq. (10) the combinatorial factor of the diagram is the order of its rotation symmetry group in the plane: for example the planar version of the diagram $\_$has combinatorial factor $\frac{1}{2}$.

The effective action of the $\lambda \phi^{4}$ theory is invariant under the following rescaling

$$
t \rightarrow \sigma t \quad \lambda \rightarrow \sigma^{-2} \lambda \quad y_{2 k} \rightarrow \sigma^{k} y_{2 k}
$$

where $\sigma$ is a real parameter. This reflects the invariance of the classical action under transformation of $t$ and $\lambda$ as in (13) and simultaneous rescaling of the classical field given by: $\phi \rightarrow \sqrt{\sigma} \phi$. Hence $H_{t}^{(+)}$is a function of the variables $\tau \equiv t^{2} \lambda$ and $\eta_{2 k} \equiv$ $t^{-k} y_{2 k}$. The equation for $\mathcal{H}\left(\tau ;\left\{\eta_{2 k}\right\}\right) \equiv H_{t}^{(+)}\left(\lambda ;\left\{y_{2 k}\right\}\right)$ is

$$
\begin{aligned}
2 \tau \partial_{\tau} \mathcal{H}= & 2 \sum_{k, k^{\prime}=1}^{\infty} k k^{\prime} \eta_{2 k+2 k^{\prime}-2} \partial_{\eta_{2 k}} \mathcal{H} \partial_{\eta_{2 k^{\prime}}} \mathcal{H} \\
& +\sum_{k=1}^{\infty} \sum_{i=0}^{2 k-2} k \eta_{2 i} \eta_{2 k-2-2 i} \partial_{\eta_{2 k}} \mathcal{H}+\sum_{k=1}^{\infty} k \eta_{2 k} \partial_{\eta_{2 k}} \mathcal{H}
\end{aligned}
$$

This form of the equation is a convenient starting point for studying the strong coupling limit of the theory, $\tau \rightarrow \infty$. In this limit the interaction term $\frac{1}{4} \tau \phi^{4}$ in the classical action dominates, so that it is natural to rescale the field as $\phi \rightarrow \tau^{-\frac{1}{4}} \phi$ and to work with the sources $\zeta_{2 k} \equiv \tau^{-\frac{k}{2}} \eta_{2 k}$. The leading term in the effective action equals $-\frac{1}{4} \log \tau-\frac{1}{2} \tau^{\frac{1}{2}} \zeta_{2}$, as it can been easily understood by considering, for example, the path integral representation of the Wilson-Polchinski action. Hence $\mathcal{H}$ admits the following asymptotic expansion for $\tau \rightarrow \infty$

$$
\begin{aligned}
\mathcal{H}\left(\tau ;\left\{\eta_{2 k}\right\}\right) & =-\frac{1}{4} \log \tau-\frac{1}{2} \tau^{\frac{1}{2}} \zeta_{2}+\widetilde{\mathcal{H}}\left(\tau ;\left\{\zeta_{2 k}\right\}\right) \\
& =-\frac{1}{4} \log \tau-\frac{1}{2} \tau^{\frac{1}{2}} \zeta_{2}+\tilde{h}_{0}\left(\left\{\zeta_{2 k}\right\}\right)+O\left(\tau^{-\frac{1}{2}}\right)
\end{aligned}
$$

The function $\tilde{h}_{0}\left(\left\{\zeta_{2 k}\right\}\right)$ is to be interpreted as the initial condition of the strong coupling limit and has to be computed by independent methods. Once $\tilde{h}_{0}\left(\left\{\zeta_{2 k}\right\}\right)$ is specified, the equation for $\widetilde{\mathcal{H}}$

$$
2 \tau \partial_{\tau} \widetilde{\mathcal{H}}=\tau^{-\frac{1}{2}}\left[2 \sum_{k, k^{\prime}=1}^{\infty} k k^{\prime} \zeta_{2 k+2 k^{\prime}-2} \partial_{\zeta_{2 k}} \widetilde{\mathcal{H}} \partial_{\zeta_{2 k^{\prime}}} \widetilde{\mathcal{H}}+\sum_{k=1}^{\infty} \sum_{i=0}^{2 k-2} k \zeta_{2 i} \zeta_{2 k-2-2 i} \partial_{\zeta_{2 k}} \widetilde{\mathcal{H}}\right]
$$

determines recursively the subleading $O\left(\tau^{-\frac{1}{2}}\right)$ terms. 


\section{Functional Derivation of Large N Wilson-Pol- chinski RG Equation}

The generating functional $\mathcal{F}(J)$ of the connected Green functions of the $N \times N$ matrix scalar theory is

$$
\mathrm{e}^{-N^{2} \mathcal{F}(J)} \equiv \mathcal{N} \int d \Phi \mathrm{e}^{-N[S(\Phi)-\operatorname{Tr} J \Phi]}
$$

where $J$ is the $N \times N$ matrix source for the quantum matrix field $\Phi$ and $S(\Phi)$ is the classical action

$$
S(\Phi)=\frac{1}{2 t} \operatorname{Tr} \Phi^{2}-\operatorname{Tr} V(\Phi)
$$

For example we can take, as in the previous section, $V(\Phi)=\frac{\lambda}{4} \Phi^{4}$. The normalization factor $\mathcal{N}$ is

$$
\mathcal{N}^{-1}=\int d \Phi \mathrm{e}^{-\frac{N}{2 t} \operatorname{Tr} \Phi^{2}}=\left(\frac{\pi t}{N}\right)^{N^{2} / 2}
$$

The Wilsonian effective action $H_{t}(\varphi)$ is the generating functional of the connected amputated Green functions. It is related to $\mathcal{F}(J)$ as follows:

$$
\mathcal{F}(J)=-H_{t}(\varphi)-\frac{1}{2 N t} \operatorname{Tr} \varphi^{2}
$$

where $J=\frac{1}{t} \varphi$.

The Wilson-Polchinski RG equation determines the dependence of $H_{t}(\varphi)$ on the "propagator" $t$. The large $N$ limit should be taken keeping the invariants

$$
y_{k} \equiv \frac{1}{N} \operatorname{Tr} \varphi^{k}
$$

where $k=1,2, \ldots$, finite as $N \rightarrow \infty$.

Taking the derivative with respect to $t$ of both sides of Eq. (17) one obtains

$$
\partial_{t} H_{t}(\varphi)-\frac{1}{2 t^{2} N} \operatorname{Tr} \varphi^{2}=-\frac{1}{2 t}+\frac{1}{2 N t^{2}}\left\langle\operatorname{Tr} \Phi^{2}\right\rangle-\frac{1}{N t^{2}}\langle\operatorname{Tr} \varphi \Phi\rangle
$$

where

$$
\langle O(\Phi)\rangle=\frac{\int d \Phi \mathrm{e}^{-N\left[S(\Phi)-\frac{1}{t} \operatorname{Tr} \varphi \Phi\right]} O(\Phi)}{\int d \Phi \mathrm{e}^{-N\left[S(\Phi)-\frac{1}{t} \operatorname{Tr} \varphi \Phi\right]}}
$$

Using

$$
\begin{aligned}
& \frac{1}{N}\langle\operatorname{Tr} \varphi \Phi\rangle=-t \operatorname{Tr} \varphi \frac{\partial \mathcal{F}}{\partial \varphi} \\
& \frac{1}{N}\left\langle\operatorname{Tr} \Phi^{2}\right\rangle=-\frac{t^{2}}{N} \operatorname{Tr} \frac{\partial^{2} \mathcal{F}}{\partial \varphi^{2}}+t^{2} N \operatorname{Tr}\left(\frac{\partial \mathcal{F}}{\partial \varphi}\right)^{2}
\end{aligned}
$$


we get

$$
\partial_{t} H_{t}=\frac{N}{2} \operatorname{Tr}\left(\frac{\partial H_{t}}{\partial \varphi}\right)^{2}+\frac{1}{2 N} \operatorname{Tr} \frac{\partial^{2} H_{t}}{\partial \varphi^{2}}
$$

which is the finite $N$ RG equation. In the large $\mathrm{N}$ limit we should keep fixed the variables $y_{k}$. Hence, let us express the derivatives with respect to $\varphi$ in terms of $y_{k}$-derivatives:

$$
\frac{\partial H_{t}}{\partial \varphi}=\sum_{k} k \frac{\varphi^{k-1}}{N} \partial_{y_{k}} H_{t}
$$

and

$$
\begin{aligned}
\frac{1}{N} \operatorname{Tr} \frac{\partial^{2} H_{t}(\varphi)}{\partial \varphi^{2}}= & \frac{1}{N^{2}} \sum_{k, k^{\prime}} k k^{\prime} y_{k+k^{\prime}-2} \partial_{y_{k}} \partial_{y_{k^{\prime}}} H_{t} \\
& +\sum_{k} \frac{k}{N^{2}} \operatorname{Tr}\left(\frac{\partial \varphi^{k-1}}{\partial \varphi}\right) \partial_{y_{k}} H_{t}
\end{aligned}
$$

In the large $N$ limit the first term in the r.h.s. of the equation above goes to zero. The second term, instead, survives. Indeed, since

$$
\operatorname{Tr}\left(\frac{\partial \varphi^{k-1}}{\partial \varphi}\right)=\sum_{i=0}^{k-2} \operatorname{Tr} \varphi^{i} \operatorname{Tr} \varphi^{k-2-i}
$$

one has

$$
\sum_{k} \frac{k}{N^{2}} \operatorname{Tr}\left(\frac{\partial \varphi^{k-1}}{\partial \varphi}\right) \partial_{y_{k}} H_{t}=\sum_{k} \sum_{i=0}^{k-2} k y_{i} y_{k-2-i} \partial_{y_{k}} H_{t}
$$

which is finite as $N \rightarrow \infty$. In conclusion the RG equation in the large $N$ limit becomes

$$
\partial_{t} H_{t}=\frac{1}{2} \sum_{k, k^{\prime}} k k^{\prime} y_{k+k^{\prime}-2} \partial_{y_{k}} H_{t} \partial_{y_{k^{\prime}}} H_{t}+\frac{1}{2} \sum_{k} \sum_{i=0}^{k-2} k y_{i} y_{k-2-i} \partial_{y_{k}} H_{t}
$$

in agreement with Eq. (\$).

\section{Application to Matrix Models}

In the application of Eq. (30) to 0-dimensional matrix models it is usually convenient to replace the $y_{k}$ variables with the eigenvalues $\xi_{i}$ of the matrix source $\varphi$. Starting from

$$
y_{k}=\frac{1}{N} \sum_{i} \xi_{i}^{k}
$$

it easily shown that

$$
\operatorname{Tr}\left(\frac{\partial H_{t}}{\partial \varphi}\right)^{2}=\sum_{i}\left(\partial_{\xi_{i}} H_{t}\right)^{2}
$$


and that

$$
\operatorname{Tr} \frac{\partial^{2} H_{t}}{\partial \varphi^{2}}=\sum_{i} \partial_{\xi_{i}}^{2} H_{t}+\sum_{i, j: i \neq j} \frac{\partial_{\xi_{i}} H_{t}-\partial_{\xi_{j}} H_{t}}{\xi_{i}-\xi_{j}}
$$

Going back to the finite $N$ RG equation (25), it rewrites therefore as follows:

$$
\partial_{t} H_{t}=\frac{N}{2} \sum_{i}\left(\partial_{\xi_{i}} H_{t}\right)^{2}+\frac{1}{2 N} \sum_{i} \partial_{\xi_{i}}^{2} H_{t}+\frac{1}{2 N} \sum_{i, j: i \neq j} \frac{\partial_{\xi_{i}} H_{t}-\partial_{\xi_{j}} H_{t}}{\xi_{i}-\xi_{j}}
$$

One can re-absorb the term linear in $\xi_{i}$ 's derivatives by defining

$$
\hat{H}_{t}=H_{t}+\frac{1}{4 N^{2}} \sum_{i, j: i \neq j} \log \left(\xi_{i}-\xi_{j}\right)^{2}
$$

Thus

$$
\partial_{t} \hat{H}_{t}=\frac{N}{2} \sum_{i}\left(\partial_{\xi_{i}} \hat{H}_{t}\right)^{2}+\frac{1}{2 N} \sum_{i} \partial_{\xi_{i}}^{2} \hat{H}_{t}
$$

In order to understand the large $N$ behaviour of the equation above, we introduce the eigenvalue density [G]

$$
\rho(\xi) \equiv \frac{1}{N} \sum_{i} \delta\left(\xi-\xi_{i}\right),
$$

and rewrite Eq. (36) as follows

$$
\partial_{t} \hat{H}_{t}=\frac{1}{2} \int d \xi \rho(\xi)\left[\left(\partial_{\xi} \frac{\delta \hat{H}_{t}}{\delta \rho(\xi)}\right)^{2}+\frac{1}{N^{2}} \partial_{\xi}^{2} \frac{\delta^{2} H_{t}}{\delta \rho(\xi)^{2}}\right]
$$

This makes clear that the second term in the r.h.s. which is second order in field derivatives is subleading in the large $N$-limit.

Thus the planar limit of the 0 -dimensional $\mathrm{RG}$ equation reduces just to the free Hamilton-Jacobi equation for a system of $N$ particles (with $N \rightarrow \infty$ )

$$
\partial_{t} \hat{H}_{t}=\frac{N}{2} \sum_{i}\left(\partial_{\xi_{i}} \hat{H}_{t}\right)^{2}
$$

We can therefore express $\hat{H}_{t}$ in terms of the initial condition

$$
\hat{V}\left(\left\{\xi_{i}\right\}\right)=\sum_{i} V\left(\xi_{i}\right)+\frac{1}{4 N} \sum_{i, j: i \neq j} \log \left(\xi_{i}-\xi_{j}\right)^{2}
$$

by means of the general integral of the free Hamilton-Jacobi equation:

$$
\hat{H}_{t}\left(\left\{\xi_{i}\right\}\right)=\frac{1}{N} \hat{V}\left(\left\{\zeta_{i}\right\}\right)-\frac{t}{2 N} \sum_{i}\left(\partial_{\zeta_{i}} \hat{V}\left(\left\{\zeta_{i}\right\}\right)\right)^{2}
$$


where the functions $\zeta_{i}\left(\xi_{i}, t\right)$ are implicitely defined by the following algebraic equation

$$
\begin{aligned}
\zeta_{i} & =\xi_{i}+t \partial_{\zeta_{i}} \hat{V}\left(\left\{\zeta_{i}\right\}\right) \\
& =\xi_{i}+t V^{\prime}\left(\zeta_{i}\right)+\frac{t}{N} \sum_{j: j \neq i} \frac{1}{\zeta_{i}-\zeta_{j}}
\end{aligned}
$$

In conclusion, we have reduced the solution of a generic matrix model with interaction $V(\Phi)$ coupled to an external source $\varphi$, in the large $N$ limit, to the solution of the algebraic equation (42). In terms of the solution $\zeta_{i}\left(\xi_{i}, t\right)$ of this equation, the large $N$ effective action $H_{t}$ of the matrix model is

$$
\begin{aligned}
H_{t} & =\frac{1}{N} \sum_{i} V\left(\zeta_{i}\right)-\frac{t}{2 N} \sum_{i}\left(V^{\prime}\left(\zeta_{i}\right)\right)^{2}-\frac{t}{2 N^{2}} \sum_{i, j: i \neq j} \frac{V^{\prime}\left(\zeta_{i}\right)-V^{\prime}\left(\zeta_{j}\right)}{\zeta_{i}-\zeta_{j}} \\
& -\frac{1}{4 N^{2}} \sum_{i, j: i \neq j} \log \left(1-t \frac{V^{\prime}\left(\zeta_{i}\right)-V^{\prime}\left(\zeta_{j}\right)}{\zeta_{i}-\zeta_{j}}+\frac{t}{N} \sum_{k: k \neq i, j} \frac{1}{\left(\zeta_{i}-\zeta_{k}\right)\left(\zeta_{j}-\zeta_{k}\right)}\right)^{2}
\end{aligned}
$$

In deriving Eq. (43) from Eqs. (35), (41) and (40) we have suppressed terms

$$
-\frac{t}{2 N^{3}} \sum_{\substack{i, j, k \\ j, k \neq i}} \frac{1}{\zeta_{i}-\zeta_{j}} \frac{1}{\zeta_{i}-\zeta_{k}}=-\frac{t}{2 N^{3}} \sum_{i, j: i \neq j} \frac{1}{\left(\zeta_{i}-\zeta_{j}\right)^{2}}
$$

and

$$
-\frac{2 t}{N} \frac{1}{\left(\zeta_{i}-\zeta_{j}\right)^{2}}
$$

because sub-leading in the $1 / N$ expansion. Note, however, that terms of order $1 / N^{p}$ with $p>0$ are still contained in equation (43).

\section{Extension to $d$ Space-Time Dimensions}

The generating functional $\mathcal{F}[J]$ of the regularized connected Green functions of the $d$-dimensional $N \times N$ matrix scalar theory is

$$
\mathrm{e}^{-N^{2} \mathcal{F}[J]} \equiv \mathcal{N} \int[d \Phi] \mathrm{e}^{-N\left[S[\Phi]-\int d^{d} x \operatorname{Tr} J(x) \Phi(x)\right]}
$$

where $J(x)$ is the $N \times N$ matrix source for the quantum matrix field $\Phi(x)$ and $S[\Phi]$ is the regularized classical action

$$
S[\Phi]=\frac{1}{2} \int d^{d} x d^{d} y \Delta_{t}^{-1}(x, y) \operatorname{Tr} \Phi(x) \Phi(y)-\int d^{d} x V(\Phi(x))
$$

As we explained in the previous section, $\Delta_{t}(x, y)$ is the propagator regulated both in the IR and in the UV. We omit explicit reference to the UV scale $t_{0}$ and denote 
by $t$ the infra-red cut-off: $\Delta_{t}(x, y)$ vanishes rapidly in the UV limit $t \rightarrow t_{0}$. The normalization factor $\mathcal{N}$ is

$$
\begin{aligned}
\mathcal{N}^{-1} & =\int[d \Phi] \mathrm{e}^{-\frac{N}{2} \int d^{d} x d^{d} y \Delta_{t}^{-1}(x, y) \operatorname{Tr} \Phi(x) \Phi(y)} \\
& =\left(\frac{\pi \operatorname{det}_{x, y}\left(\Delta_{t}(x, y)\right)}{N}\right)^{N^{2} / 2}
\end{aligned}
$$

The Wilsonian effective action $H_{t}[\varphi]$ is related to $\mathcal{F}[J]$ as follows:

$$
\mathcal{F}[J]=-H_{t}[\varphi]-\frac{1}{2 N} \int d^{d} x d^{d} y \Delta_{t}^{-1}(x, y) \operatorname{Tr} \varphi(x) \varphi(y)
$$

where $J(x)=\int d^{d} y \Delta_{t}^{-1}(x, y) \varphi(y)$.

The Wilson-Polchinski RG equation determines the dependence of $H_{t}[\varphi]$ on the infra-red scale $t$. The large $N$ limit should be taken by keeping the invariants

$$
Y_{k}\left(x_{1}, \ldots, x_{k}\right) \equiv \frac{1}{N} \operatorname{Tr} \varphi\left(x_{1}\right) \cdots \varphi\left(x_{k}\right)
$$

where $k=1,2, \ldots$, finite as $N \rightarrow \infty$. Going through the same steps as in the previous section, we obtain the finite $N$ RG equation

$$
\partial_{t} H_{t}=\frac{N}{2} \int d^{d} x d^{d} y \dot{\Delta}_{t}(x, y)\left[\operatorname{Tr} \frac{\delta H_{t}}{\delta \varphi(x)} \frac{\delta H_{t}}{\delta \varphi(y)}+\frac{1}{N^{2}} \operatorname{Tr} \frac{\delta^{2} H_{t}}{\delta \varphi(x) \delta \varphi(y)}\right]
$$

To take the large $N$ limit we must express the derivatives with respect to $\varphi$ in terms of $Y_{k}$-derivatives:

$$
\frac{\delta H_{t}}{\delta \varphi(x)}=\sum_{k} \frac{k}{N} \int d x_{1} \ldots d x_{k-1} \varphi\left(x_{1}\right) \cdots \varphi\left(x_{k-1}\right) \frac{\delta H_{t}}{\delta Y_{k}\left(x, x_{1}, \ldots, x_{k-1}\right)}
$$

For the same reasons that have been explained in the previous section, the second derivative term in Eq. (51) reduces, in the large $N$ limit, to

$$
\begin{aligned}
& \frac{1}{N} \operatorname{Tr} \frac{\delta^{2} H_{t}}{\delta \varphi(x) \delta \varphi(y)} \rightarrow \sum_{k} \sum_{i=1}^{k-1} k \int d x_{1} \ldots d x_{k-1} \delta\left(x_{i}-x\right) \\
& \quad \times Y_{i-1}\left(x_{1}, \ldots, x_{i-1}\right) Y_{k-1-i}\left(x_{i+1}, \ldots, x_{k-1}\right) \frac{\delta H_{t}}{\delta Y_{k}\left(y, x_{1}, \ldots, x_{k-1}\right)}
\end{aligned}
$$

In conclusion the RG equation in the large $N$ limit becomes

$$
\begin{aligned}
\partial_{t} H_{t}=\frac{1}{2} \int d x d y \dot{\Delta}_{t}(x, y)\left[\sum_{k, k^{\prime}} k k^{\prime} \int d x_{1} \ldots d x_{k-1} d y_{1} \ldots d y_{k^{\prime}-1}\right. \\
\times Y_{k+k^{\prime}-2}\left(x_{1}, \ldots, x_{k-1}, y_{1}, \ldots, y_{k^{\prime}-1}\right)
\end{aligned}
$$




$$
\begin{aligned}
& \times \frac{\delta H_{t}}{\delta Y_{k}\left(x, x_{1}, \ldots, x_{k-1}\right)} \frac{\delta H_{t}}{\delta Y_{k^{\prime}}\left(y, y_{1}, \ldots, y_{k^{\prime}-1}\right)}+ \\
& +\sum_{k} \sum_{i=1}^{k-1} k \int d x_{1} \ldots d x_{k-1} \delta\left(x_{i}-x\right) \\
& \times Y_{i-1}\left(x_{1}, \ldots, x_{i-1}\right) Y_{k-1-i}\left(x_{i+1}, \ldots, x_{k-1}\right) \\
& \left.\times \frac{\delta H_{t}}{\delta Y_{k}\left(y, x_{1}, \ldots, x_{k-1}\right)}\right]
\end{aligned}
$$

This is still a Hamilton-Jacobi type equation for the system of multi-local sources $Y_{k}\left(x_{1}, \ldots, x_{k}\right)$. Due to the non-trivial $t$-dependence of $\dot{\Delta}_{t}(x, y)$, this system is described by a time-dependent Hamiltonian, in contrast to the zero-dimensional case. Note that, even if we derived Eq. (53) in the framework of $N \times N$ matrix theory, the equation is valid for the non-commutative Moyal case as well.

\section{Conclusions}

In this article we have derived, both from diagrammatic and functional methods, the "planar" Wilson-Polchinski RG equation for a $d$-dimensional scalar theory with fields valued in a generic non-commutative algebra. We have emphasized that a closed RG equation exists for the functional generator of connected amputated graphs with sphere topology and all possible configurations of external legs, which represent multi-traces of the field sources. The crucial difference between the ordinary and the planar Wilson-Polchinski equations lies in the quantum (loop) term: it is proportional to second order field derivatives in the ordinary equation and to first order field derivatives in the planar one. Thus the planar RG equation for a $d$-dimensional system has the form of a Hamilton-Jacobi equation for a non-relativistic system in $d+1$ dimensions, whose time is identified with the renormalization scale.

This result is of course reminiscent of the holographic conjecture. However a precise relation - if it exists at all - between our $d$-dimensional RG equation and the Hamilton-Jacobi equation for $d+1$-dimensional supergravity of [8] remains unclear to us. In particular, it should be stressed that Wilson RG, even in Polchinski's differential form, describes an invariance property of the Feynman integral and hence concerns the effective action which is a functional of the elementary fields while the object that enters the holographic conjecture is a vacuum energy, functional of the sources of composite operators.

The planar Wilson-Polchinski equation should be a useful tool to investigate the renormalization properties of field theories over non-commutative space. The equation

describes the regime when external momenta are large in comparison with $1 / \sqrt{|\Theta|}$, with $\Theta$ the non-commutative space-time tensor, and thus it does not concern the $|\Theta| \rightarrow 0$ limit. As first explained in [4], this limit is made subtle by the fact that 
non-planar Green functions contains negative powers of $\Theta$. This does not necessarily imply the presence of singularities of the renormalized theory in the limit $|\Theta| \rightarrow 0$. Indeed the limit of small non-commutativity should be studied by renormalizing the theory at an energy scale is much smaller than $1 / \sqrt{|\Theta|}$. In this framework deformations corresponding to the small $|\Theta|$ expansion of the star product should represent irrelevant terms. From this point of view the UV/IR paradox just originates from a not-allowed limit exchange.

As a last application, we have shown that in the case of 0-dimensional hermitian matrix models the large $N$ RG equation can be recast in a very simple form: it is equivalent to the free Hamilton-Jacobi equation for a system of particles in 1 dimension. This reformulation has lead to a closed expression for the large $N$ effective action of the model, which is equivalent to the free energy of one-matrix model coupled to an external matrix source. As far as we know, the expression in Eqs. (43), (42) for the free energy of this model with arbitrary potential represents a new result.

\section{Acknowledgments}

We thank M. Porrati for helpful discussions. This work is supported in part by the European Commission's Human Potential programme under contract HPRNCT-2000-00131 "The quantum structure of spacetime and the geometric nature of fundamental interactions", to which the authors are associated through the Frascati National Laboratory, and by Ministero dell'Università e della Ricerca Scientifica e Tecnologica.

\section{A Non-Planar Non-Commutative Graphs}

To understand how one can approach a field theory over a non-commutative spacetime using our RG equation in the framework of perturbation theory it can be useful to present the first steps of the construction.

The starting point is the introduction of a cut-off propagator that we choose according to:

$$
\Delta_{\Lambda}\left(p^{2}\right)=\int_{\Lambda_{0}^{-2}}^{\Lambda^{-2}} d x \mathrm{e}^{-x p^{2}}
$$

Here $\Lambda_{0}$ is the UV cut-off and $\Lambda$ is the running cut-off that is identified with the RG

parameter. Let $1 / \sqrt{|\Theta|}$ be the non-commutative mass scale. We will consider the evolution equation in the regime

$$
\frac{1}{\sqrt{|\Theta|}}<\Lambda \ll \Lambda_{0}
$$


The effective action $H_{\Lambda}[\varphi]$ is computed as a series expansion of the coupling $\lambda$ starting from the initial condition

$$
H_{\Lambda_{0}}[\varphi]=\frac{\lambda}{4} \int \prod_{i=1}^{4} d p_{4, i} \delta\left(\sum_{j=1}^{4} p_{4, j}\right)\left\langle\varphi\left(p_{4,1}\right) \cdots \varphi\left(p_{4,4}\right)\right\rangle \mathrm{e}^{i \Phi\left(p_{4,1} \ldots p_{4,4}\right)}
$$

where we have defined the Moyal factor

$$
\Phi\left(p_{k, 1} \ldots p_{k, k}\right) \equiv \sum_{i=2}^{k-1}\left(\sum_{j=1}^{i-1} p_{k, j}\right) \Theta p_{k, i}
$$

The terms in the effective action of Eq. (3) proportional to the amplitudes $h_{\Lambda}^{\left\{\delta_{k, 2}\right\}}\left(p_{k, j_{k}}^{\left(i_{k}\right)}\right)$ and $h_{\Lambda}^{\left\{\delta_{k, 6}\right\}}$ are determined at the first perturbative order to be:

$$
\begin{aligned}
& \lambda \int \prod_{i=1}^{2} d p_{2, i} \delta\left(\sum_{j=1}^{2} p_{2, j}\right)\left[\int \frac{d p}{(2 \pi)^{d}} \int_{\Lambda_{0}^{-2}}^{\Lambda^{-2}} d x \mathrm{e}^{-x p^{2}}+C\right]\left\langle\varphi\left(p_{2,1}\right) \varphi\left(p_{2,2}\right)\right\rangle \\
& +\frac{\lambda^{2}}{2} \int \prod_{i=1}^{6} d p_{6, i} \int d p \delta\left(\sum_{j=1}^{3} p_{6, j}+p\right) \delta\left(\sum_{l=4}^{6} p_{6, l}-p\right) \int_{\Lambda_{0}^{-2}}^{\Lambda^{-2}} d x \mathrm{e}^{-x p^{2}} \\
& \times \mathrm{e}^{i \Phi\left(p_{6,1} \cdots p_{6,6}\right)}\left\langle\varphi\left(p_{6,1}\right) \cdots \varphi\left(p_{6,6}\right)\right\rangle
\end{aligned}
$$

These contributions correspond, in the zero dimensional case, to the terms linear in $t$ in Eq. (12). The constant $C$ in the first term of equation above appears due to the UV divergence of the corresponding diagram; it must be determined using suitable normalization conditions at some fixed $\Lambda_{R}>1 / \sqrt{|\Theta|}$. For example, if we require $h_{\Lambda}^{\left\{\delta_{k, 2}\right\}}$ to vanish at $p_{2,1}=0$ at the normalization point $\Lambda_{R}$, the term in brackets $\int \frac{d p}{(2 \pi)^{d}} \int_{\Lambda_{0}^{-2}}^{\Lambda^{-2}} d x \mathrm{e}^{-x p^{2}}+C$ becomes $(4 \pi)^{-\frac{d}{2}}\left(1-\frac{d}{2}\right)^{-1}\left(\Lambda^{d-2}-\Lambda_{R}^{d-2}\right)$, which is UV safe.

With a further step we reach the terms corresponding to the $t^{2}$ contributions in (12). At this order the first non-planar term in the perturbative expansion of the effective action appears:

$$
\int \prod_{i_{2}, j_{2}=1}^{2} d p_{2, j_{2}}^{\left(i_{2}\right)} \delta\left(\sum_{i_{2}, j_{2}=1}^{2} p_{2, j_{2}}^{\left(i_{2}\right)}\right) h_{\Lambda}^{\left\{2 \delta_{k, 2}\right\}}\left(p_{k, j_{k}}^{\left(i_{k}\right)}\right)\left\langle\varphi\left(p_{2,1}^{(1)}\right) \varphi\left(p_{2,2}^{(1)}\right)\right\rangle\left\langle\varphi\left(p_{2,1}^{(2)}\right) \varphi\left(p_{2,2}^{(2)}\right)\right\rangle
$$

The non-planar amplitude $h_{\Lambda}^{\left\{2 \delta_{k, 2}\right\}}\left(p_{k, j_{k}}^{\left(i_{k}\right)}\right)$ receives contributions fron the two following diagrams (with inward oriented momenta):
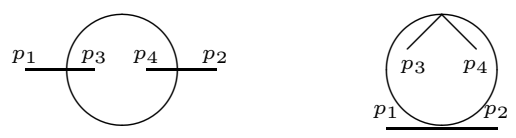
Let us consider only the first diagram whose contribution to $h_{\Lambda}^{\left\{2 \delta_{k, 2}\right\}}$ is:

$$
\begin{aligned}
& h_{\Lambda, 1}^{\left\{2 \delta_{k, 2}\right\}}=\frac{\lambda^{2}}{4} \int \frac{d k}{(2 \pi)^{d}} \mathrm{e}^{i 2 k \Theta\left(p_{1}+p_{2}\right)} \int_{\Lambda_{0}^{-2}}^{\Lambda^{-2}} d x d y \mathrm{e}^{-\left[x\left(k+\frac{p_{2}+p_{4}}{2}\right)^{2}+y\left(k-\frac{p_{2}+p_{4}}{2}\right)^{2}\right]} \\
& =\frac{\lambda^{2}}{4(4 \pi)^{\frac{d}{2}}} \int_{\Lambda_{0}^{-2}}^{\Lambda^{-2}} \frac{d x d y}{(x+y)^{\frac{d}{2}}} \mathrm{e}^{-\frac{1}{(x+y)}\left[x y\left(p_{2}+p_{4}\right)^{2}+\left(\Theta\left(p_{1}+p_{2}\right)\right)^{2}+i(x-y)\left(p_{2}+p_{4}\right) \Theta\left(p_{1}+p_{2}\right)\right]}
\end{aligned}
$$

In the non-exceptional regime, in which

$$
\frac{1}{\sqrt{|\Theta|}}<\Lambda<\left|p_{1}+p_{2}\right| \sim\left|p_{2}+p_{4}\right|
$$

one can safely take the (UV) limit $\Lambda_{0} \rightarrow \infty$ and, after a variable rescaling, one obtains

$$
\begin{aligned}
h_{\Lambda, 1}^{\left\{2 \delta_{k, 2}\right\}} & =\frac{\lambda^{2}}{4(4 \pi)^{\frac{d}{2}}|\Theta|^{\frac{d}{2}-2}} \int_{0}^{\frac{1}{|\Theta| \Lambda^{2}}} \frac{d x d y}{(x+y)^{\frac{d}{2}}} \\
& \times \mathrm{e}^{-\frac{1}{(x+y)}\left[x y|\Theta|\left(p_{2}+p_{4}\right)^{2}+\frac{\left(\Theta\left(p_{1}+p_{2}\right)\right)^{2}}{|\Theta|}+i(x-y)\left(p_{2}+p_{4}\right) \Theta\left(p_{1}+p_{2}\right)\right]}
\end{aligned}
$$

Hence asymptotically, that is when $\frac{1}{\sqrt{|\Theta|}}<\Lambda \ll\left|p_{1}+p_{2}\right| \sim\left|p_{2}+p_{4}\right|$, we find

$$
\begin{aligned}
h_{\Lambda, 1}^{\left\{2 \delta_{k, 2}\right\}} & \simeq \frac{\lambda^{2}}{4(4 \pi)^{\frac{d}{2}}|\Theta|^{\frac{d}{2}-2}} \int_{0}^{\frac{1}{|\Theta| \Lambda^{2}}} \frac{d x d y}{(x+y)^{\frac{d}{2}}} \mathrm{e}^{-\frac{1}{(x+y)} \frac{\left(\Theta\left(p_{1}+p_{2}\right)\right)^{2}}{|\Theta|}} \\
& \simeq \frac{\lambda^{2}}{4(4 \pi)^{\frac{d}{2}}\left(\frac{\Lambda^{2}}{2}\right)^{2-\frac{d}{2}}} \int_{1}^{2} d z z^{\frac{d}{2}-2} \mathrm{e}^{-\left(\frac{1}{2} \Theta\left(p_{1}+p_{2}\right)\right)^{2} \Lambda^{2} z} \\
& \simeq \frac{\lambda^{2}}{4(4 \pi)^{\frac{d}{2}}\left(\frac{\Lambda^{2}}{2}\right)^{2-\frac{d}{2}}} \mathrm{e}^{-\left(\frac{1}{2} \Theta\left(p_{1}+p_{2}\right)\right)^{2} \Lambda^{2}}
\end{aligned}
$$

This example confirms that when external momenta are non-exceptional non planar amplitudes are exponentially suppressed as noted in the Introduction.

One could wonder how it is possible that non-planar terms contribute to the evolution equation of planar ones - the observation of which fact is the main result of this paper. Our example clarifies also this point. Indeed the hole amplitude that we have studied contributes to the evolution equation of the two-loop self energy by a term in which two legs belonging to the same hole are connected by $\dot{\Delta}$. However and this is the crucial remark - this situation does not belong to the non-exceptional regime since $p_{3}+p_{4}=-p_{1}-p_{2}=0$. In this case it is apparent that the exponential damping factor in (63) disappears. 


\section{B Differential Equations for Matrix Models}

Let $F\left(\left\{g_{n}\right\}\right)$ be the vacuum energy of 0 -dimensional matrix model with an infinite number of single-trace interactions

$$
\mathrm{e}^{N^{2} F\left(\left\{g_{n}\right\}\right)} \equiv \int d \Phi \mathrm{e}^{N S_{1}\left(\Phi ;\left\{g_{n}\right\}\right)}
$$

where

$$
S_{1}\left(\Phi ;\left\{g_{n}\right\}\right)=\sum_{n=1}^{\infty} g_{n} \operatorname{Tr} \Phi^{n}
$$

The scale invariance of the matrix functional measure implies that

$$
e^{-N^{2} F} \int d \Phi \operatorname{Tr} \partial_{\Phi}\left(\Phi \mathrm{e}^{N S_{1}}\right)=0
$$

which translates in the following first order differential equation for $F\left(\left\{g_{n}\right\}\right)$

$$
1+\sum_{n=1}^{\infty} n g_{n} \partial_{g_{n}} F=0
$$

A similar first order linear differential equation follows from translation invariance of the functional measure. In an analogous way we can derive a non-linear differential equation for $F\left(\left\{g_{n}\right\}\right)$ starting from

$$
e^{-N^{2} F} \int d \Phi \operatorname{Tr} \partial_{\Phi}^{2} \mathrm{e}^{N S_{1}}=N\left\langle\operatorname{Tr} \partial_{\Phi}^{2} S_{1}\right\rangle+N^{2}\left\langle\operatorname{Tr}\left(\partial_{\Phi} S_{1}\right)^{2}\right\rangle=0
$$

Using the relations

$$
\begin{aligned}
& \left\langle\operatorname{Tr}\left(\partial_{\Phi} S_{1}\right)^{2}\right\rangle=N \sum_{n, m=1}^{\infty} n m g_{n} g_{m} \partial_{g_{n+m-2}} F \\
& \left\langle\operatorname{Tr} \partial_{\Phi}^{2} S_{1}\right\rangle=N^{2} \sum_{n=1}^{\infty} \sum_{l=0}^{n-2} n g_{n}\left(\partial_{g_{l}} F \partial_{g_{n-2-l}} F+\frac{1}{N^{2}} \partial_{g_{l}} \partial_{g_{n-2-l}} F\right)
\end{aligned}
$$

(and reorganizing the sums) we find, in the large $N$ limit

$$
\sum_{n, m=0}^{\infty}(n+m+2) g_{n+m+2} \partial_{g_{n}} F \partial_{g_{m}} F+\sum_{n=0}^{\infty} \sum_{l=1}^{n+1} l(n-l+2) g_{l} g_{n-l+2} \partial_{g_{n}} F=0
$$

where we define $\partial_{g_{0}} F \equiv 1$.

Eq. (70) allows to recursively determine $F\left(\left\{g_{n}\right\}\right)$ as a perturbative series in $g_{2}^{-1}$. In order to make the recursive computation simple it is convenient to introduce the following functional

$$
\hat{F}_{t}\left(\left\{g_{n}\right\}\right) \equiv-\frac{1}{2} \log t+F\left(\left\{g_{n}-\frac{1}{2 t} \delta_{n, 2}\right\}\right)
$$


which is essentially the functional considered in [10]. More precisely, the functional of [10] coincides with $\hat{F}_{t}\left(\left\{g_{n}\right\}\right)$ after the couplings $g_{n}$ with $n \leq 2$ have been set to zero. Note however that a simple differential equation can only be written for $\hat{F}_{t}\left(\left\{g_{n}\right\}\right)$ as a functional of all the couplings $g_{n}$ with $n \geq 1$. The definition Eq. (71) and the two differential equations (67) and (71) yield

$$
\partial_{t} \hat{F}_{t}=\frac{1}{2} \sum_{n, m=0}^{\infty}(n+m+2) g_{n+m+2} \partial_{g_{n}} \hat{F} \partial_{g_{m}} \hat{F}+\frac{1}{2} \sum_{n=0}^{\infty} \sum_{l=1}^{n+1} l(n-l+2) g_{l} g_{n-l+2} \partial_{g_{n}} \hat{F}
$$

which is the equation derived in [10]. Eq. (72) and the initial condition

$$
\left.\hat{F}_{t}\left(\left\{g_{n}\right\}\right)\right|_{t=0}=0
$$

determine $\hat{F}_{t}\left(\left\{g_{n}\right\}\right)$ as a power series in $t$. The first terms of the series are

$$
\hat{F}_{t}=\left(g_{2}+\frac{1}{2} g_{1}^{2}\right) t+\left(g_{2}^{2}+g_{1}^{2} g_{2}+\frac{3}{2} g_{1} g_{3}+\frac{3}{2} g_{6}+\frac{5}{2} g_{1} g_{5}+g_{1}^{2} g_{4}\right) t^{2}+O\left(t^{3}\right)
$$

\section{References}

[1] G. 't Hooft, "A Planar Diagram Theory For Strong Interactions," Nucl. Phys. B 72 (1974) 461.

[2] J. Maldacena, "The large $N$ limit of superconformal field theories and supergravity," Adv. Theor. Math. Phys. 2 (1998) 231, hep-th/9711200.

[3] E .Witten, "Anti-de Sitter space and holography," Adv. Theor. Math. Phys. 2 (1998) 253, hep-th/9802150.

[4] S. Minwalla, M. Van Raamsdonk and N. Seiberg, "Noncommutative perturbative dynamics," JHEP 0002 (2000) 020, hep-th/9912072;

M. Van Raamsdonk and N. Seiberg, "Comments on noncommutative perturbative dynamics," JHEP 0003 (2000) 035, hep-th/0002186.

[5] M. R. Douglas and N. A. Nekrasov, "Noncommutative field theory," Rev. Mod. Phys. 73 (2002) 977, hep-th/0106048.

[6] K. G. Wilson and J. B. Kogut, "The Renormalization Group And The Epsilon Expansion," Phys. Rept. 12 (1974) 75.

[7] J. Polchinski, "Renormalization And Effective Lagrangians," Nucl. Phys. B 231 (1984) 269. 
[8] J. de Boer, E. Verlinde and H. Verlinde, "On the holographic renormalization group," JHEP 0008 (2000) 003, hep-th/9912012;

E. Verlinde and H. Verlinde, "RG-flow, gravity and the cosmological constant," JHEP 0005 (2000) 034, hep-th/9912018;

J. Khoury and H. Verlinde, "On open/closed string duality," Adv. Theor. Math. Phys. 3 (1999) 1893, hep-th/0001056;

J. de Boer, "The holographic renormalization group," Fortsch. Phys. 49 (2001) 339 , hep-th/0101026.

[9] E. Brezin, C. Itzykson, G. Parisi and J. B. Zuber, "Planar Diagrams," Commun. Math. Phys. 59 (1978) 35.

[10] M. Li, "A note on relation between holographic RG equation and Polchinski's RG equation," Nucl. Phys. B 579 (2000) 525, hep-th/0001193. 